\title{
Infant-mother attachment and the growth of externalizing problems across the primary-school years
}

Article

Accepted Version

Fearon, R. M. P. and Belsky, J. (2011) Infant-mother attachment and the growth of externalizing problems across the primary-school years. Journal of Child Psychology and Psychiatry, 52 (7). pp. 782-791. ISSN 1469-7619 doi: https://doi.org/10.1111/j.1469-7610.2010.02350.x Available at https://centaur.reading.ac.uk/17504/

It is advisable to refer to the publisher's version if you intend to cite from the work. See Guidance on citing.

To link to this article DOI: http://dx.doi.org/10.1111/j.1469-7610.2010.02350.x

Publisher: Wiley-Blackwell

All outputs in CentAUR are protected by Intellectual Property Rights law, including copyright law. Copyright and IPR is retained by the creators or other copyright holders. Terms and conditions for use of this material are defined in the End User Agreement.

www.reading.ac.uk/centaur 
Central Archive at the University of Reading

Reading's research outputs online 
Infant-Mother Attachment and the Growth of Externalizing Problems Across the Primary-School Years

\author{
R.M. Pasco Fearon \\ University of Reading \\ and \\ Jay Belsky \\ Birkbeck University of London
}

WORD COUNT $=7963$ 


\begin{abstract}
Background: Some contend that attachment insecurity increases risk for the development of externalizing behavior problems in children. Method: Latent-growth curve analyses were applied to data on 1364 children from the NICHD Study of Early Child Care to evaluate the association between early attachment and teacher-rated externalizing problems across the primary-school years. Results: Findings indicate that (a) both avoidant and disorganized attachment predict higher levels of externalizing problems but (b) that effects of disorganized attachment are moderated by family cumulative contextual risk, child gender and child age, (c) with disorganized boys from risky social contexts manifesting increases in behavior problems over time. Conclusions: These findings highlight the potentially conditional role of early attachment in children's externalizing behavior problems and the need for further research evaluating causation and mediating mechanisms.
\end{abstract}

Keywords: ATTACHMENT, EXTERNALIZING PROBLEMS, CUMULATIVE

RISK, GENDER. 
Infant-Mother Attachment and the Growth of Externalizing Problems Across the Primary-School Years

Attachment refers to a special facet of the affectional bonds between young children and their caregivers wherein the child selectively seeks out the parent in times of stress as a means of achieving comfort and feelings of safety. The developmental sequelae of individual differences in the patterning of attachment behavior in infancy, as observed in laboratory tasks involving separation from and reunion with a primary caregiver, have been a focus of investigation for decades (Matas, Arend \& Sroufe, 1978). Many developmentalists contend that a secure attachment pattern - characterised by proximity seeking behaviour during separation and reunion, and, when contact is achieved, an effective calming and return to play carries with it developmental advantages that persist over time and influence socioemotional functioning and risk for disorder (Kobak et al., 2005). Two 'insecure' patterns of attachment, Avoidant and Disorganized, have emerged in longitudinal studies as the most prominent potential markers of risk for later childhood externalizing behaviour problems (Renken, Egeland, Marvinney, Mangelsdorf et al., 1989; Van IJzendoorn, Schuengel \& Bakermans-Kranenburg, 1999). Avoidant attachment is characterized by a muted expression of attachment behaviour during separation, and avoidance of the caregiver during reunion, whereas Disorganization reflects an apparent disruption in the organization or coherence of attachment behaviour, as demarcated by behaviors such as sudden stilling, disorientation and rocking (Main \& Goldwyn, 1990).

Results of several longitudinal and cross-sectional studies have revealed these two patterns of attachment to be associated with elevated levels of externalizing behavior problems (e.g. Lyons-Ruth, Alpern \& Repacholi, 1993; Moss, Cyr \& 
Dubois-Comtois, 2004; Renken et al., 1995). For example, in one of the earliest and most important longitudinal studies of the consequences of attachment security and insecurity, avoidant attachment was found to be related to higher teacher-rated externalizing problems at age 4.5 years (Erikson, Egeland \& Sroufe, 1985). In a later follow-up of this sample at grades 1-3 avoidant attachment continued to predict higher externalizing problems, although at this age only in boys (Renken et al., 1989).

Similarly, several longitudinal studies have found that infants classified as disorganized evidence higher levels of behavior problems in childhood (Lyons-Ruth et al., 1993; Moss, et al., 2004), and a meta-analysis of 12 studies indicated that this effect is relatively consistent and of medium effect size (Van IJzendoorn, et al., 1999). Nevertheless, questions remain as to whether, the extent to which, and the conditions under which, attachment avoidance or disorganization in infancy forecast future behavioural or emotional problems. Both positive and negative findings have been reported in the literature and a recent meta-analysis found substantial heterogeneity in effect sizes across studies that had related security and disorganization to children's externalizing problems (Fearon, Bakermans-Kranenburg, Van IJzendoorn, Lapsley \& Roisman, 2010). In this paper, we examine for the first time three critical factors that may modulate the predictive significance of disorganized and avoidant attachment for later externalizing problems: 1) the degree of family social-contextual risk, 2) the age at which outcome is measured, and 3) child gender. In particular, we test the idea that the effects of disorganized attachment increase, rather than diminish, with time, and do so primarily for boys growing up in conditions of relative adversity. Furthermore, we extend our inquiry by testing the degree to which continuities between infancy attachment and later externalizing problems can be explained by maternal sensitivity occurring in infancy, or subsequently, during the school years. 
Variation in the level of family adversity is a plausible factor that might explain inconsistencies in the apparent predictive significance of attachment, as past research seems to have been most likely to discern a predictive association between insecurity and problem behavior when it has involved high contextual-risk samples (e.g. Erickson et al., 1985). Belsky and Fearon (2002) set out to explicitly test this possibility using problem-behavior data collected at age 36 months in the large-scale NICHD Study of Early Child Care and Youth Development (SECCYD) (NICHD Early Child Care Research Network [ECCRN], 2005). Consistent with the claim that insecure attachment functions as a risk factor that predicts problem behaviour in interaction with cumulative social-contextual risk, they found that insecure attachment forecasted 36 month mother-reported externalizing problems more strongly when contextual risk was pronounced. The current study seeks to extend this work by examining the degree to which attachment and contextual risk interact in predicting externalizing problems right across the primary-school years. In addition, by using teacher reports rather than maternal reports, we limit, if not eliminate, possible common bias in the measurement of attachment and outcome.

An additional finding of potential importance from this earlier study of nearly 1,000 children was that insecure-avoidance in particular predicted externalizing problems, not insecure-resistance or disorganization. Whereas the lack of an association involving resistance was perhaps not surprising, the absence of links involving disorganisation, even at the highest levels of contextual risk, was unexpected. At the time, Belsky and Fearon (2002) speculated that the effects of disorganization might emerge later in development, as the majority of extant outcome studies implicating disorganized attachment in children's behavior problems involved school-aged children, not toddlers or preschoolers. This proposition received some 
indirect support in a recent meta-analysis, which revealed that, in the case of studies using the SSP--the majority of the 69 samples meta-analyzed--effect sizes relating attachment to externalizing problems tended to be larger when the outcome was measured later in development (Fearon et al., 2010). However, in this large set of studies the insecure categories of resistant, avoidant and disorganised attachment were not distinguished, so the contribution of each to the effect discerned could not be determined. Nevertheless, in an analysis of a subset of published papers that reported separate outcome statistics for individual insecure attachment groups, disorganization emerged as more strongly associated with externalizing problems than the other insecure categories. These meta-analytic findings could be regarded as consistent, then, with the hypothesis that the effects of disorganization emerge more strongly over time. However, the meta-analysis proved inconclusive on this point, as no clear relationship emerged between age at outcome assessment and disorganized attachment within this smaller set of studies. Another limit of the meta-analytic data was that it was based on comparisons of single data points (one outcome measurement) compared across studies, rather than repeated measurements within studies, and so even the findings regarding overall security could only provide indirect support for the prospective hypothesis that attachment effects on externalizing problems emerge more strongly with development.

More convincing evidence for the proposal that risk-elevating effects of attachment insecurity - and possibly disorganized attachment in particular - take time to emerge, requires repeated longitudinal follow up. This is what the current inquiry offers. Indeed, not only is the present study positioned to examine relations between early attachment, including disorganization, and later externalizing problems, but it does so using annual teacher reports of such behavior across primary-school Grades 
1-6 so that the growth of externalizing problems can be investigated. In addition, the current report addresses a further important issue highlighted by several studies (e.g. Renken et al., 1995), narrative reviews (e.g. Kobak et al., 2005) and by Fearon et al.'s (2010) meta-analysis, namely, whether gender moderates the effect of attachment on externalizing problems. In line with Renken et al.'s (1989) earlier findings, Fearon et al. (2010) discerned substantially stronger meta-analytic associations between child behavior problems and attachment in samples consisting only of boys compared to those with only girls, both at the level of overall security, and specifically in relation to disorganized attachment.

In addition to addressing the moderating effects of contextual risk, age and gender, the current report examines one further lacuna highlighted by several authors, the possibility that attachment effects may be accounted for by the quality of concurrent parenting in infancy or may be carried by continuity in parenting occurring beyond the infancy period (Lamb, Thompson, Gardner, Charnov \& Estes, 1984). Thus, after determining whether and how early attachment predicts externalizing problems during middle childhood, the current inquiry evaluates whether prediction holds once the sensitivity of parenting in infancy is statistically controlled and whether longitudinal prediction is accounted for by sensitive mothering occurring during the school years.

\section{Method}

\section{Participants}

The NICHD Study of Early Child Care and Youth Development (SECCYD) recruited 1364 families through hospital visits shortly after the birth of a child in 1991 at 10 U.S. locations (for detailed description of recruitment procedures and sample 
characteristics see NICHD ECCRN, 2001). During selected 24-hour intervals, all women giving birth $(N=8,986)$ were screened for eligibility. From that group, 1364 families completed a home interview when the infant was 1 month old and became the study participants. Details of the sampling plan can be found in NICHD ECCRN, 2005). In terms of demographic characteristics, $26 \%$ of the mothers had no more than a high school education at time of enrollment; $21 \%$ had incomes no greater than $200 \%$ of the poverty level at $6^{\text {th }}$ grade; and $22 \%$ were minority (i.e., not non-Hispanic European American).

As with any longitudinal study, not all families participated in every wave of data collection. Relatively few families formally withdrew $\left(\mathrm{N}=291\right.$ through $6^{\text {th }}$ grade), but almost all children had some missing data. Children were least likely to be missing direct assessments and most likely to be missing teacher ratings. Of the 1364 children, 248 had no teacher rated outcome data at all, and 561 had teacher data at every single outcome point (Grades 1 through 6 ). $75 \%$ of the sample had three or more outcome measurements. Children with complete data tended to be from families with a higher income $(t(1354)=4.24, p<.001$; mean income to needs ratio $=4.0$, S.D. $=2.66$ versus 3.31, S.D. $=3.11)$ and to have parents who provided more responsive care in infancy $(t(1146)=3.97, p<.001)$ than did children with missing data. Children with missing data were also more likely to come from single parent families $(t(1362)=3.17, p=.002)$, and there were significant differences in the proportion of families from minority ethnic communities with complete data, compared to those with some missing data $\left(\chi^{2}(2)=34.5, p<.001\right)$. In order to obtain estimates of effects that were not biased by excluding cases with missing data, all 1364 cases were included in the analyses, which employed Full Information Maximum Likelihood estimation. The Full Information Maximum Likelihood approach makes use of all 
available data and estimates optimal parameter estimates when data are missing at random (Allison, 2003).

\section{Measures}

Measurements are described in terms of their roles in the analyses to be reported: attachment predictor, cumulative-contextual-risk moderator, teacherreported externalizing outcome and maternal-sensitivity covariate. Information about this public data set can be found at http://secc.rti.org/. Ethical approval was granted by each of the 10 data-collecting universities before any data included in this reported was collected and at each age of measurement informed consent was secured from parents and/or teachers.

\section{Attachment Security}

Infant-mother attachment security was assessed at 15 months using the Ainsworth and Wittig (1969) Strange Situation procedure. Each SSP videotape was coded twice at a central location by two of three coders blind to all information on the children, using the standard classifications of secure (B), insecure-avoidant (A), insecure-resistant (C), disorganized (D), and unclassifiable (U). Across all coder pairs, agreement with the 5-category classification system was 83\% (kappa= .69) (NICHD ECCRN, 1997). Only cases classified A, B, C, or D are included in this study $(N=1149)$.

\section{Cumulative Contextual Risk}

To create an index of cumulative contextual risk, emphasis was placed on empirically robust and objective indicators, summing the total number present in each family out of four. Economic Risk was present when the average income-to-needs ratio across 11 measurement occasions from 6 months of age through Grade 6 fell below the $20^{\text {th }}$ percentile for the sample The mean income to needs ratio for this risk 
group was $.70(S . D .=.41)$, where a value of 1 or less reflects poverty status. Father Absence Risk was defined in terms of the number of times (out of 11) that mother reported not living with the child's father. When this value exceeded $60 \%$, which it did for $19.6 \%$ of the sample $(N=367)$, father absence risk was designated. Education Risk occurred whenever mothers failed to graduate from high school (i.e., 12 years of schooling); $10.2 \%$ of mothers $(N=139)$ met this criterion. Age Risk was designated in $12.2 \%$ of the sample $(N=166)$ for those mothers who gave birth to the target child at age 20 or younger.

Cumulative risk scores ranged from $0-4 ; 67.2 \%(N=911)$ received scores of zero, $14.8 \%(N=201)$ scores of $1,9.7 \%(N=131)$ scores of 2 , and $8.3 \%(N=112)$ scores of 3 or 4 . Cumulative risk was treated as an ordered linear, rather than categorical/nominal, variable, thereby maximizing statistical power while avoiding problems of estimating interaction effects with large degrees of freedom and excessively small cell sizes.

\section{Teacher-reported Externalizing Problems}

The Child Behavior Checklist Teacher Report Form (Achenbach, 1991) was used to evaluate problem behavior. The TRF lists 100 problem behaviors that generate two subscales: Internalizing Problems (e.g., "too fearful and anxious") and Externalizing Problems (e.g., "hits others", “disobedient at school”, "argues a lot”). Achenbach reports test-retest reliability of .89 , interparent agreement of .70 , and stability of .71 over two years. Raw scores were used in order to capture absolute growth in the teacher-reported means over time.

\section{Maternal Sensitivity}

Measures of maternal behavior were collected repeatedly using videotaped mother-child interactions. Those obtained in infancy (15 months), at $1^{\text {st }}, 3^{\text {rd }}$ and $5^{\text {th }}$ 
grade were used in this study, reflecting times corresponding to key ages in this research. The interaction tasks changed over time, providing a means of assessing age-appropriate qualities of maternal behavior.

The observation task at 15 months involved the three-boxes procedure, in which mother shows the child experimenter-provided toys in three containers in a set order (see Vandell, 1979). At $1^{\text {st }}$-grade, tasks included working together to draw a picture of a house and a tree using an Etch-A-Sketch (with each person controlling one knob), a patterned-block activity using colored blocks of different parquet shapes to fill in geometric frames, and a card game. Two activities were used in $3^{\text {rd }}$ and $5^{\text {th }}$ grades, the first at each time being a discussion of topics that were sources of disagreement between the mother and child and the second being a challenging dyadic planning task (for details, see Belsky et al., 2007).

All videotapes were shipped to a central location for coding, one different from that used to code SSP videotapes. Teams of coders blind to information on the family scored the mother-child interaction videotapes at each time period, with one or two members of a coding team carrying over from one age period to the next. Coders received intensive training and supervision; complete operational and coding manuals can be found at http://secc.rti.org/. Composite maternal sensitivity scores at 15 months were created from the sums of 34 -point ratings (maternal sensitivity to child nondistress, intrusiveness [reversed], and positive regard. At older ages, the maternalsensitivity composite reflected the sum of 3 7-point ratings (supportive presence, respect for autonomy and hostility[reversed]). Inter-coder reliability was calculated as the intra-class correlation coefficient. Reliability for the composite scores exceeded .83 at every age. Cronbach alphas exceeded .70 at every age. We selected the sensitivity scores for 15 months and grade 1 and the average of $3 \mathrm{rd}^{\text {rd }}$ and $5^{\text {th }}$ grade 
sensitivity scores so that controls could be employed for sensitivity measured around the time of the attachment assessment (15 months), at the time of the starting point for outcome assessment (i.e. at Grade 1 - the time point corresponding to the intercept in the latent growth curve analyses) and covering a period approximately spanning the timeframe of potential change subsequent to grade 1 (the average of Grades 3 and 5 sensitivity).

\section{Data analysis}

We analyzed the longitudinal data using latent growth curve (LGC) models, which represent a multivariate approach to longitudinal data analysis based on the general structural equation modeling framework (Curran \& Willoughby, 2003). In addition to allowing for the estimation of overall growth and heterogeneity in growth curves at the individual level, the approach allows for the flexible analysis of a range of other analytic issues, such as the invariance of growth parameters across groups, the influence of constant and time-varying covariates and the efficient estimation of effects in the presence of missing data (Curran \& Willoughby, 2003).

The models were configured to estimate the initial level (intercept) and growth (linear and quadratic curves) in externalizing problems from Grades 1 to 6 for each child, as well as the overall averages and inter-individual variances of these growth parameters using maximum likelihood estimation. The quality of model fit (how well the statistical model reproduced the observed data) was tested using the maximumlikelihood ratio test statistic and indices of model fit (the Comparative Fit Index [CFI] and the Root Mean Square Error of Approximation [RMSEA]). CFI values of .90 or higher and RMSEA values of .08 or lower are generally considered to indicate acceptable model fit (Bentler, 1990). We also examined the extent to which the same pattern of growth obtained across genders, by comparing models where the growth 
parameters were free to vary between boys and girls with one in which they were constrained to be equal. Having established the adequacy of the LGC models, we then tested the effects of attachment (dummy variable coded) and risk on the growth of externalizing problems by including them as regressors on the intercept and slopes for externalizing problems. Attachment-x-risk interactions were specified by multiplying risk by three dummy variables representing each insecure attachment category (A versus non-A, D versus non-D, C versus non-C) and comparing the fit and difference in the likelihood ratio test statistic of nested models where these terms were free to be estimated with models where they were constrained to be zero. Finally, three-way interactions involving attachment, risk and gender were tested by comparing a model in which the attachment-x-risk interactions were fixed to be equal in boys and girls with one in which these two-way interactions were free to vary across genders, following procedures described by Curran, Bauer and Willoughby (2004). The extent to which any identified effects of attachment remained after controlling for sensitivity was addressed by including the three sensitivity variables (15 months, grade 1 , average of grades 3 and 5) as additional regressors in the above models. Because there was a considerable amount of missing data, and because excluding such cases can undermine statistical power and bias parameter estimates (Allison, 2003), we employed the Full Information Maximum Likelihood (FIML) method, which uses all the available data to estimate the parameter estimates of a model (by calculating the log-likelihood of the data for each observational unit separately). This approach is clearly superior to mean substitution and listwise deletion, and of comparable performance to multiple imputation (Allison, 2003; Schafer \& Graham, 2002). All analyses were conducted using Mplus version 4.21 (Muthen \& Muthen, 2006). 
The results are presented in X sections: 1) descriptive statistics, 2) testing the adequacy of the basic growth curve model for teacher-reported externalizing problems from grades 1 to 6,3 ) testing main effects of attachment on these growth curves, 4) testing attachment-X-Risk-X-gender interactions, 5) the role of maternal sensitivity, and 6) testing whether specific risk indicators were responsible for the discerned interactions between cumulative risk and attachment.

Descriptive statistics and latent growth curve modelling of teacher data

Table 1 provides descriptive statistics for teacher-reported externalizing problems from Grades 1-6, as well as the covariances/correlations among them. The data show that teacher-reported externalizing problems peak in Grade 3, declining thereafter, suggesting that a curvilinear model might best characterize the overall pattern of growth. A latent-growth curve model with random intercept, linear slope and quadratic slope that allowed the intercept and slope means to differ between girls and boys fit the data fairly well $\left(\chi^{2}(27)=89.79, p<.001 ; \mathrm{CFI}=.97, \mathrm{RMSEA}=.062\right)$, with significant inter-individual variance in all three growth parameters (Intercept Variance $=40.67, S E=3.43 ;$ Linear Slope Variance $=9.75, S E=1.72 ;$ Quadratic Slope Variance $=0.36, S E=.062$ ). Despite the relatively good fit of this model, allowing the variances of the growth parameters to be different in boys and girls led to a significant improvement in fit $\left(\Delta \chi^{2}(3)=18.17, p<.001\right.$; Model fit: $\chi^{2}(24)=71.62, p$ $<.001 ; \mathrm{CFI}=.98, \mathrm{RMSEA}=.060)$. Accordingly, in all the analyses that follow the intercepts and slopes (means and variances) were estimated separately for boy and girls.

Table 2 presents the sample means and standard deviations of teacher ratings of externalizing problems from grade 1 to 6 separately by attachment classification, gender and two levels of risk (grouped into 0/1 vs. 2+ risks for ease of presentation). 
In the next three sections we formally test the contribution of 1) attachment, 2) attachment $\mathrm{x}$ risk interactions and 3) their modulation by gender on the growth of externalizing problems over time using latent growth curve modelling.

\section{Attachment main effects on latent growth curve parameters}

The first step involved estimating latent-growth curve models that included only main effects of attachment on the intercept and change parameters of the latent growth model for children's externalizing problems. This approach was adopted for two reasons--to facilitate comparisons with other studies that have not considered contextual risk when evaluating effects of attachment and to obtain estimates of effect sizes unadjusted for contextual risk for the purpose of facilitating interpretation of subsequent results. We began by testing a LGC for teacher reports of externalizing problems with attachment dummy variables specified as predictors of the LGC intercept and slopes. In this initial model we constrained the attachment effects to be the same in boys and girls. This model fit the data well $\left(\chi^{2}(51)=93.47, p<.001\right.$; $\mathrm{CFI}=.98, \mathrm{RMSEA}=.037$ ). When the attachment effects were free to vary between boys and girls the model did not significantly improve in fit $\left(\Delta \chi^{2}(9)=7.64, p=.57\right)$. Significant reductions in model fit occurred when the attachment effects on the intercept $\left(\Delta \chi^{2}(3)=11.26, p=.010\right)$ and slopes $\left(\Delta \chi^{2}(6)=18.42, p=.005\right)$ were forced to be zero, indicating significant effects of attachment on the average teacher reports of externalizing problems at Grade 1 and in the change in these reports over time. The parameter estimates for the attachment effects are shown in Table 3. As can be seen in the table, avoidance (relative to security) was associated with a higher intercept ( $B=$ 2.43, se $=.74, \beta=.13, p=.001)$, indicating that infants classified as avoidant in infancy were rated as having more externalizing problems at Grade 1 (the intercept) than their secure counterparts. The effect size for this difference between avoidant and 
secure infants was $d=.35$. Re-estimation of the model with the intercept specified to indicate later grades showed that this effect was also significant at Grade $2(z=2.39$, $d=.21, p=.017$ ), but not at later grades. At grade 1 , the avoidant children also scored higher than the resistant $(z=2.24, d=.35, p=.024)$, but not disorganized, children $(\mathrm{z}=1.73, d=.23, p=.084)$.

There was also a significant effect of disorganization on the quadratic slope. In order to interpret this effect, the predicted growth curves were plotted for each attachment category (see Figure 1). Inspection of Figure 1 indicates that the trajectory for disorganized children demonstrated curvature, with an increasing trajectory after approximately grade 3 . This led to differences between secure and disorganised children that were significant by grades $5(z=2.21, d=.17, p=.027)$ and $6(z=3.72$, $d=.28, p<.001)$. The disorganized group also scored more highly at these two points in time than the resistant group $(z=2.21, d=.24, p=.027 ; z=2.54, d=.30, p=.011$, respectively), although these differences were of marginal statistical significance.

The effect of avoidance on the intercept and disorganization on the quadratic slope remained significant when contextual risk was controlled $(B=1.77$, se $=.52$ and $B=.26, s e=.096$ respectively). NOTE: SIGNIGFICANCE THRESHOLD NOT CONSISTENTLY APPLIED $(\mathrm{P}<.01)$ ABOVE.

\section{Attachment $x$ contextual risk interactions}

To address the study's primary hypotheses, terms representing attachment-Xlinear risk interactions were added as predictors of the intercept and growth parameters of the LGC model for children's externalizing problems. This model, initially with risk effects and attachment-X-risk interactions fixed to be equal across males and females fit the data well, $\chi^{2}(87)=194.62, p<.001 ; \mathrm{CFI}=.96$, RMSEA $=.043$. Allowing the effect of risk to vary for males and females led to a 
significant improvement in model fit $\left(\Delta \chi^{2}(3)=19.32, \mathrm{p}<.001\right)$, with males demonstrating a significantly larger effect of risk on the intercept than females (though not the slopes). Furthermore, allowing the attachment-X-risk effects on the intercept and slopes to vary as a function of gender significantly improved model fit $\left(\Delta \chi^{2}(9)=28.26, p<.001\right)$, indicating significant gender-X-attachment-X-risk interactions. In order to identify the nature of these interactions, we estimated the effects of attachment, risk and their interaction separately for boys and girls. In each case we tested the increase in model fit when attachment-X-risk interactions were added to a model that only had main effects (strictly, simple main effects for each gender), beginning with their effects on the intercept, then linear slope and finally quadratic slope. For boys, adding attachment-X-risk interaction effects on the intercept did not lead to a significant increase in model fit $\left(\Delta \chi^{2}(3)=6.67, p=.083\right)$. However, when attachment-X-risk interactions were included for the linear slope, the model fit increased significantly $\left(\Delta \chi^{2}(3)=22.03, p<.0001\right)$. The parameter estimates for these attachment-X-risk interactions are shown in Table 3. As can be seen in the table, for boys there was a highly significant and positive disorganization-X-risk interaction effect on the linear slope $(B=1.22$, se $=.28, \beta=.13, p<.0001)$, indicating greater risk-related increases in externalizing problems in disorganized boys over time, relative to secure boys. The risk interactions involving avoidance and resistance were not significant at the $1 \%$ level. Finally, when attachment-X-risk interaction effects on the quadratic slope were added to the model, no further significant increases in model fit were observed $\left(\Delta \chi^{2}(3)=6.88, p=.076\right)$.

To facilitate interpretation of the disorganization-X-risk interaction on the linear slope for boys, predicted growth curves were plotted for each attachment category at two levels of risk (zero and 2 risks). This plot is shown in Figure 2. As the 
chart clearly shows, the disorganized boys at the higher level of risk showed quite marked increases in externalizing problems between grades 1 and 6 , peaking at grade 5. Consistent with this interpretation, at the level of 2 risks there was a highly significant effect of disorganization on the linear slope $(B=1.84, \beta=.16$, se $=.47, p$ $<.001)$. At this higher level of risk, disorganized boys scored significantly higher than the secure and avoidant boys at grade 5 (D versus B mean difference $=6.51$, se $=$ $1.80, d=.80, p<.001 ; \mathrm{D}$ versus A mean difference $=6.99, s e=2.73, d=1.02, p=$ $.001)$ and Grade 6 (D versus B mean difference $=7.53, s e=2.29, d=.82, p=.001 ; \mathrm{D}$ versus A mean difference $=6.33, s e=2.73, d=.77, p=.010$ ). These same differences were also significant at grade 4, whereas at grade 3 the disorganized boys scored more highly than avoidant (mean difference 5.99, se $=2.21, d=.73, p=.006$ ) and resistant (mean difference $=7.68, s e=2.94, d=.93, p=.008$ ), but not secure, boys (mean difference 3.31, se $=1.85, d=.40, p=.073$ ). At the low level of risk, there were no significant attachment effects on the intercept or slopes.

For girls, none of the risk-X-attachment effects were significant for the intercept or slopes. There was a weak main effect of avoidance on the intercept $\left(\Delta \chi^{2}(1)=5.76, p=.016\right)$ and slopes $\left(\Delta \chi^{2}(2)=7.21, p=.027\right)$ and a weak effect of disorganization on the slopes $\left(\Delta \chi^{2}(2)=7.92, p=.019\right)$. As none of these effects was significant at the $1 \%$ level, we did not pursue them any further.

It should be noted that these analyses focussed on the growth of continuously distributed externalizing scores, not rates of clinical disorder. In this relatively low risk sample, only a small number of children scored in the clinical range ( $\mathrm{T}$ score $=>64$ ). To illustrate, amongst boys at high contextual risk, only 16 scored in the clinical range at grade 4, of whom 6 were disorganized in infancy $(O R=3.8, C I 1.01-$ 14.35). At grade 5, 17 scored in the clinical range, of whom 9 were disorganized in 
infancy $(O R=15.38 C I 3.40-69.62)$. At grade 6, 22 scored in the clinical range, of whom 7 were disorganized in infancy $(O R=2.60$, CI 0.74-8.96).

The role of maternal sensitivity

Additional analyses determined whether the discerned attachment effects could be accounted for by differences in maternal sensitivity. The latent growth models were thus re-estimated, controlling for sensitivity effects on the baseline and slope parameters where appropriate. There were essentially two principal effects that had arisen from the previous analyses - an overall main effect of avoidance on the intercept and a risk-X-disorganization effect on the slopes for boys. When the attachment main effects model was re-estimated, controlling for the sensitivity variables (15 months and Grade 1), the effect of avoidance was substantially reduced and became non-significant ( $B$ in previous analysis was 2.43 ; with sensitivity controlled $B=1.31, s e=.723, p=.070)$. To test the significance of the reduction in the effect of avoidance, we conducted tests of indirect effects, based on bootstrap methods described by Preacher and Hayes (2008). These analyses indicated that sensitivity at 15 months and grade 1 both accounted for significant portions of the avoidance effect on the intercept, although grade 1 sensitivity made the larger contribution (indirect effect $=.38, s e=.14 ; 15$ months sensitivity indirect effect $=.10$, $s e=.06)$

In a parallel manner, we re-estimated the attachment-X-risk-X-gender model (with effects of attachment, risk and their interaction estimated separately for boys and girls), with sensitivity at 15 months, grade 1 and the average of grades 3 and 5 included as covariates on the intercept and slopes. In contrast to the results for avoidance, no appreciable change emerged in the previously detected disorganizationX-risk interaction on the boys' linear slope when sensitivity was controlled $(B=1.25$, 
$s e=.28, p<.001 ;$ in previous analysis without sensitivity controlled $B=1.21, s e=$ $.28, p<.001)$

Decomposing risk effects

Finally, we sought to determine whether the interactive effect of disorganization and risk on the trajectories of boys' behaviour problems could be isolated to particular risk indicators, or indeed whether the overall effect might be larger when separate effects of each indicator were used instead of a single cumulative risk variable. To minimize the number of statistical tests conducted, we focused only on the effects of attachment disorganization on the linear slope among boys. When all four risk-X-disorganization interaction effects on the slope were constrained to be zero, the reduction in model fit was significant $\left(\Delta \chi^{2}(8)=20.08, p=\right.$ .009). Notably however, there was limited independent predictive power from the individual risk indicators; all independent effects of the singular-risk-Xdisorganization interactions were non-significant at the $1 \%$ level. The disorganization$\mathrm{X}$-low income interaction was significant at the $5 \%$ level $(B=2.06$, se $=.958, p=$ .030). The original model using the cumulative risk index accounted for $7 \%$ of the total variance in the linear slope for males, of which $1.45 \%$ was attributable to the disorganization-X-risk interaction. In contrast, when separate and single risk indicators were entered, $16 \%$ of the total variance in the linear slope was accounted for, of which $1.7 \%$ was attributable to disorganization-X-risk interactions (of which there were four). The interaction effects involving attachment were thus broadly comparable in size, particularly given that the variance accounted for inevitably increases when more variables are included in the model.

Discussion 
Many contend that early attachment security is important for children's mental health. Yet uncertainty remains regarding the extent to which common variations in attachment are associated with behaviour problems, let alone whether more stringent empirical criteria for inferring causation can be met (Rutter, 2004). Carefully controlled, longitudinal studies have found evidence that both avoidant and disorganized attachment may be implicated in the development of externalizing problems in childhood (Renken et al., 1995; Lyons-Ruth et al., 1993). However, not all studies have found such associations, and the insecure attachment patterns that have emerged as being linked with children's externalizing problems have not been entirely consistent.

The present research sought to test whether early attachment security predicted later externalizing problems and, notably, their growth over time in middle childhood, taking advantage of a large longitudinal dataset. Three critical unresolved issues were addressed: (a) whether children with insecure-avoidant or insecure-disorganized attachment histories manifest elevated levels of externalising problems; (b) whether effects of insecure attachment, and disorganized attachment in particular, strengthen over time; and (c) whether effects of attachment insecurity (disorganization or avoidance) on externalising problems are amplified under conditions of high contextual risk. Also examined was whether the aforementioned effects were stronger amongst boys.

Longitudinal analyses revealed significant associations between attachment and school-aged children's behavior problems, and these effects were not reducible to social-contextual risk; they were either independent of it (in the case of avoidance) or interacted with it (in the case of disorganization). The data also proved consistent with the claim that attachment may evince stronger associations with children's 
development under conditions of contextual risk (Belsky \& Fearon, 2002). Notable, though, was that the discerned interactions between attachment and risk were only evident in children classified as disorganized in infancy, which contrasts with earlier findings from the same sample when outcomes were measured at age 3 (Belsky \& Fearon, 2002). Then it was primarily children (1) with insecure-avoidant histories (2) who lived in conditions of high risk, who manifested elevated levels of externalizing problems. Indeed, at age 3 there was no evidence of poorer functioning for children who had been classified as disorganized in infancy at all, measured across several developmental domains (e.g., language, mother-reported behavior problems). This difference in findings could be due to the different reporters of problems - mothers vs. teachers - between the two studies or the different developmental periods investigated. The latter possibility finds some corroboration in the growth curve findings reported here. Recall that they revealed a minimal effect of disorganization on externalizing problems in Grade 1, but a steeper rate of growth in problems thereafter, such that disorganized boys in conditions of multiple contextual risks scored higher than all children in all other contexts by Grades 5-6. Indeed, by Grade 6 there was a difference of 7.5 points on the raw externalizing scores for these children relative to secure boys at the same level of contextual risk, which is a large effect size $(d=.82)$.

The results just summarised are striking because they align two apparently contradictory findings. First, as noted already, it may help explain why Fearon and Belsky (2002) failed to detect anticipated effects of disorganization at age 3 in the NICHD Study. Second, it is consistent with Fearon et al.'s (2010) meta-analysis showing that attachment insecurity in the SSP was associated more consistently with externalizing problems when externalizing problems were assessed at older than at younger ages. Whereas the meta-analysis could not identify which insecure group was 
responsible for this result, the current findings suggest that it may be attributable to disorganized children, particularly those living under conditions of high contextual risk.

Finally, in keeping with the view that disorganization is not strongly related to parenting sensitivity (Van IJzendoorn, Schuengel \& Bakermans-Kranenburg., 1999), the effects for disorganization remained when maternal sensitivity in infancy and in middle-childhood were controlled. In contrast, the main effect of avoidance on the externalizing intercept term was reduced substantially when sensitivity was controlled. This suggests significant overlap between these two effects, as might have been predicted (Lamb et al., 1984). Furthermore, the findings pointed to the potential importance of the subsequent quality of care for some of the longitudinal effects of avoidant attachment on later externalizing problems.

In sum, the current findings provide further evidence that individual differences in attachment behavior are associated with differences in children's behavior problems in the school years. They further indicate that the effects of attachment disorganization in particular may increase over time, especially for boys under conditions of high contextual adversity. The findings provide further indication that disorganized attachment may play a significant role in clinical problems with aggression and conduct, but the generally low-risk nature of this sample and the low rates of clinical disorder observed within it, caution against making strong direct inferences of this nature from the current findings alone.

Despite the notable results reported, there is an urgent need for research capable of addressing competing explanations for the hypothesised attachment effects chronicled here, including continuities in environmental third variables or genetic factors. Furthermore, the field would benefit enormously from improvements in 
measurement protocols for attachment that would allow rigorous use of longitudinal cross-lagged designs in order to address the causal status of the association between attachment and externalizing behavior problems. Intervention trials with long-term follow-up, ideally conducted in high-risk populations, could yield valuable information on this critical issue, while also advancing the science and practice of prevention. 


\section{References}

Achenbach, T. M. (1991). Manual for the Teacher's Report Form and 1991 Profile.

Burlington, VT: University of Vermont Department of Psychiatry.

Allison, P.D. (2003). Missing data techniques for structural equation modeling. Journal of Abnormal Psychology, 112, 545-557.

Belsky, J., \& Fearon, R. M. P. (2002). Infant-mother attachment security, contextual risk, and early development: A moderational analysis. Development and Psychopathology. Vol, 14(2), 293-310.

Belsky, J., Vandell, D. L., Burchinal, M., Clarke-Stewart, K. A., McCartney, K., \& Owen, M. T. (2007). Are there long-term effects of early child care? Child Development, 78, 681-701.

Bentler, P. M. (1990). Comparative fit indices in structural models. Psychological Bulletin, 107, 238-246.

Curran, P.J. \& Willoughby, M.T. (2003). Implications of latent trajectory models for the study of developmental psychopathology. Development and Psychopathology, 15, 581-612.

Curran, P.J., Bauer, D.J. \& Willoughby, M.T. (2004). Testing main effects and interactions in latent curve analysis. Psychological Methods, 9, 220-237.

Fearon, R.M. P., Bakermans-Kranenburg, M.J., Van IJzendoorn, M.H., Lapsley, A. \& Roisman (2010). The significance of insecure attachment and disorganization in the development of children's externalizing behavior: A meta-analytic study. Child Development, 81, 435-456.

Kobak, R., Cassidy, J., Lyons Ruth, K., \& Ziv, Y. (2005). Attachment, stress and psychopathology: a developmental pathways model. In D. Cicchetti \& J. D. Cohen (Eds.), Developmental Psychopathology, Volume I: Theory and Method (2nd ed., pp. 333-369). Hoboken, NJ: Wiley. 
Lamb, M. E., Thompson, R. A., Gardner, W. P., Charnov, E. L., \& Estes, D. (1984). Security of infantile attachment as assessed in the "strange situation": Its study and biological interpretation. Behavioral and Brain Sciences, 7, 127-172.

Lyons Ruth, K., Alpern, L., \& Repacholi, B. (1993). Disorganized infant attachment classification and maternal psychosocial problems as predictors of hostile-aggressive behavior in the preschool classroom. Child Development, 64(2), 572-585.

Matas, L., Arend, R. A., \& Sroufe, L. A. (1978). Continuity of adaptation in the second year: The relationship between quality of attachment and later competent functioning. Child Development, 49, 547-556.

Moss, E., Cyr, C., \& Dubois-Comtois, K. (2004). Attachment at Early School Age and Developmental Risk: Examining Family Contexts and Behavior Problems of Controlling-Caregiving, Controlling-Punitive, and Behaviorally Disorganized Children. Developmental Psychology, 40(4), 519-532.

NICHD Early Child Care Network. (1997). The effects of infant child care on infantmother attachment security: Results of the NICHD Study of Early Child Care. Child Development, 68, 860-879.

NICHD Early Child Care Research Network. (2005). Child Care and Child Development: Results of the NICHD Study of Early Child Care and Youth Development. New York: Guilford Press.

NICHD Early Child Care Research Network (2006). Infant-mother attachment classification: risk and protection in relation to changing maternal caregiving quality over time. Developmental Psychology, 42, 38-58.

Preacher, K. J., \& Hayes, A. F. (2008). Asymptotic and resampling strategies for assessing and comparing indirect effects in multiple mediator models. Behavior Research Methods, 40, 879-891. 
Renken, B., Egeland, B., Marvinney, D., Mangelsdorf, S., \& et al. (1989). Early childhood antecedents of aggression and passive-withdrawal in early elementary school. Journal of Personality, 57, 257-281.

Rutter, M. (2004). Environmentally-mediated risks for psychopathology: research strategies and findings. Journal of the American Academy of Child and Adolescent Psychiatry, 44, $3-18$.

Schafer, J.L. \& Graham, J.W. (2002). Missing data: our view of the state of the art. Psychological Methods, 7, 147-177.

Vandell, D. L. (1979). Effects of a playgroup experience on mother-son and fatherson interaction. Developmental Psychology, 15, 379-385.

Van IJzendoorn, M.H., Schuengel, C., \& Bakermans-Kranenburg, M.J. (1999). Disorganized attachment in early childhood: Meta-analysis of precursors, concomitants, and sequelae. Development and Psychopathology, 11, 225-249. 
Table 1 Correlation/Covariance Matrices, Means and S.D.s for Teacher Reports of Externalizing Problems from Grade 1 through Grade 6

\begin{tabular}{lcccccc}
\hline & Grade 1 & Grade 2 & Grade 3 & Grade 4 & Grade 5 & Grade 6 \\
\hline Grade 1 & 66.83 & 0.56 & 0.52 & 0.52 & 0.46 & 0.52 \\
Grade 2 & 39.13 & 73.12 & 0.61 & 0.57 & 0.49 & 0.44 \\
Grade 3 & 39.58 & 49.15 & 87.88 & 0.63 & 0.52 & 0.52 \\
Grade 4 & 38.32 & 43.74 & 52.97 & 80.11 & 0.58 & 0.55 \\
Grade 5 & 34.72 & 38.20 & 44.54 & 47.88 & 84.44 & 0.58 \\
Grade 6 & 39.03 & 34.59 & 44.63 & 44.95 & 48.78 & 84.19 \\
Mean & 5.63 & 5.63 & 6.47 & 5.70 & 6.07 & 5.55 \\
S.D. & 8.18 & 8.55 & 9.37 & 8.95 & 9.19 & 9.18 \\
Ns & $1008-784$ & $921-733$ & $982-781$ & $914-758$ & $927-785$ & $855-733$ \\
\hline Note: Variances are presented on the diagonal. Below the diagonal are covariances, \\
above the diagonal are correlations.
\end{tabular}

